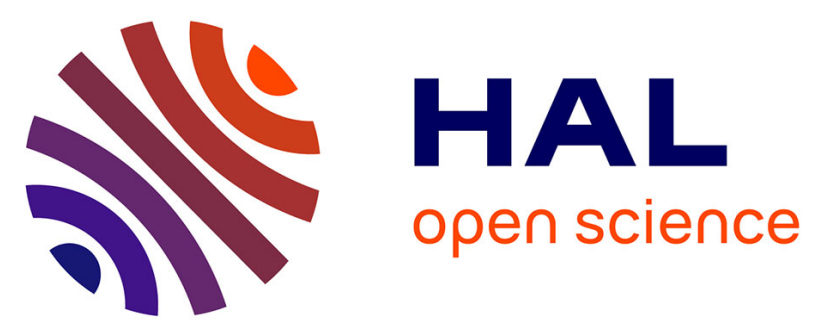

\title{
Characterization of the charge-carrier transport properties of IIa-Tech SC diamond for radiation detection applications
}

\author{
M. Pomorski, Colin Delfaure, N. Vaissiere, H. Bensalah, J. Barjon, M.-A. \\ Pinault-Thaury, D. Tromson, P. Bergonzo
}

\section{To cite this version:}

M. Pomorski, Colin Delfaure, N. Vaissiere, H. Bensalah, J. Barjon, et al.. Characterization of the charge-carrier transport properties of IIa-Tech SC diamond for radiation detection applications. physica status solidi (a), 2015, 212 (11), pp.2553 - 2558. 10.1002/pssa.201532230 . hal-01864863

\section{HAL Id: hal-01864863 https://hal.science/hal-01864863}

Submitted on 16 Jan 2020

HAL is a multi-disciplinary open access archive for the deposit and dissemination of scientific research documents, whether they are published or not. The documents may come from teaching and research institutions in France or abroad, or from public or private research centers.
L'archive ouverte pluridisciplinaire HAL, est destinée au dépôt et à la diffusion de documents scientifiques de niveau recherche, publiés ou non, émanant des établissements d'enseignement et de recherche français ou étrangers, des laboratoires publics ou privés. 


\title{
Characterization of the charge-carrier transport properties of Ila-Tech SC diamond for radiation detection applications
}

\author{
M. Pomorski', C. Delfaure', N. Vaissiere², H. Bensalah², J. Barjon², M.-A. Pinault-Thaury², D. Tromson', \\ and P. Bergonzo,
}

${ }^{1}$ CEA, LIST, Diamond Sensors Laboratory, 91191 Gif-sur-Yvette, France

${ }^{2}$ Groupe d'Etude de la Matière Condensée (GEMaC), Centre National de la Recherche Scientifique (CNRS),

Université de Versailles Saint Quentin en Yvelines (UVSQ), 45 avenue des Etats Unis, 78035 Versailles Cedex, France

Received 29 March 2015, revised 8 June 2015, accepted 17 June 2015

Published online 17 July 2015

Keywords chemical vapor deposition, crystals, diamond, electronic transport, radiation detectors

*Corresponding author: e-mail philippe.bergonzo@cea.fr, Phone: +3316908 40 53, Fax: +331 69081819

\begin{abstract}
Single crystal (SC) diamond has since years demonstrated its interest for the fabrication of radiation detectors, especially where the material properties are providing superior interests with respect to the detection application. Among the industrial suppliers able to provide on a commercial basis high-grade single crystal diamond, IIa-Tech has recently appeared in the market as a new player. The aim of this paper is to assess the quality of one SC sample when characterized under $\alpha$-particles for the measurement of its carrier transport properties. We observed that full charge collection could be observed at biases as low as $0.11 \mathrm{~V} / \mu \mathrm{m}$ with no space charge
\end{abstract}

build-up (conventionally typical bias values used are closer to $1 \mathrm{~V} / \mu \mathrm{m})$. Velocity reached values of $38 \mu \mathrm{m} / \mathrm{ns}$ and $53 \mu \mathrm{m} / \mathrm{ns}$ for electrons and holes, respectively (values probed at $0.33 \mathrm{~V} / \mu \mathrm{m}$ ). Similarly, the $\alpha$ detection spectrum displays a sharp line demonstrating the good uniformity of the material over its surface. By combining the measurements with more conventional optical observations such as birefringence and cathodoluminescence spectroscopy, it comes that the material demonstrates its ability to be used as a detector, with properties that can compare with the highest grade materials today available on the market.
1 Introduction Ultra high purity single crystal CVD (scCVD) diamond enables the fabrication of devices for a variety of radiation detection applications ranging from high energy physics to medical or X-ray beam dosimetry, profile or monitoring, and for nuclear contamination detection as well as nuclear production plant monitoring [1-4]. Several studies have demonstrated how diamond can offer superior properties to its competitor material for such applications, and including fast charge collection, harsh environment resilience, low leakage current, all at room temperatures as well as in hot environments or in corrosive media.

Among the available forms of diamond, single crystal solely exhibits the low defect densities needed for such applications. So far, only one commercial supplier had managed to fabricate high-quality materials to the community over the last years, a material that over took all its competitors by far, such as no one was ever capable to match the performances observed [5-7]. More recently, a new supplier has appeared, namely IIa-Tech, offering scCVD on the market. Open questions have risen with respect to the material performances in terms of electronic and transport properties. Here, we have used one IIa-Tech single crystal sample of $\sim 900 \mu \mathrm{m}$ in thickness for the fabrication of one ionization chamber that enabled the measurement of the transport properties in diamond. Following optical primary characterization, we have used an $\alpha$-particle-induced transient current technique (TCT) approach that allows to probe the charge carriers drift velocity as a function of the applied electric field, the saturation velocity, the charge collection, the effective trapping time, and to observe the effective space charge during electron or hole drift. This approach, further to probing directly any space charge limited effects, also enables to evaluate the long-term stability of the transport properties, due to charge accumulation as well as trapping. 


\section{Experimental}

2.1 Sample The sample under study is a high purity intrinsic diamond $<100>$ oriented, grown from the CVD technique and delivered by IIa-Technologies Singapore [8]. Lateral size of the sample is $3 \times 3 \mathrm{~mm}^{2}$ and its thickness is of $890 \mu \mathrm{m}$ (Fig. 1). Birefringence image obtained using cross polarizers shows a couple of point defects to the right of the sample, although a major part (left of the picture) shows no dislocation, a remarkable feature for a sample of such a thickness. For electrical characterization, surfaces of the sample are cleaned in hot acid solution (saturated $\mathrm{H}_{2} \mathrm{SO}_{4}$ $+\mathrm{KNO}_{3}$ solution at $290{ }^{\circ} \mathrm{C}$ during $20 \mathrm{~min}$ ), rinsed in DI water, dried and then plated with $\sim 200 \mathrm{~nm}$ thick $\mathrm{Al}$ contacts by physical deposition (magnetron sputtering) in parallel plate geometry using shadow masks.

2.2 Cathodoluminescence The single crystal diamond was analyzed by cathodoluminescence (CL) spectroscopy to evaluate its optical quality and purity. The CL spectrum was performed under a $10 \mathrm{keV}$ electron beam in a JEOL7001F scanning electron microscope (SEM) at the GEMaC laboratory. The stopping depth of electrons in diamond is then about $900 \mathrm{~nm}$. The e-beam current was fixed to $10 \mathrm{nA}$, measured with a Faraday cup. The single crystal diamond was coated with a semitransparent gold layer $(50 \AA)$ in order to conduct away electrical charges. The sample was cooled down at $8 \mathrm{~K}$ thanks to a GATAN cold finger using liquid helium. A high sensitivity UV setup from Horiba Jobin Yvon SAS was used to analyze the CL emission. The light was collected by a parabolic mirror and injected via an achromatic optical bench into a TRIAX550 monochromator equipped with a 1800 groves/ $\mathrm{mm}$ diffraction grating and an UV enhanced-silicon CCD camera. The ultimate spectral resolution is $0.02 \mathrm{~nm}$. The CL equipment allows to analyze the emission in the 200 $1000 \mathrm{~nm}$ range.

2.3 TCT (transient current technique) To perform the TCT measurements, we use a spectroscopic grade Am241 radioactive source (5.486 $\mathrm{MeV} \alpha$-particles) as a generator of excess charge carriers within the diamond bulk. Metalized diamond sample is mounted in a screened Al box, clamped between two glass-ceramic printed circuit

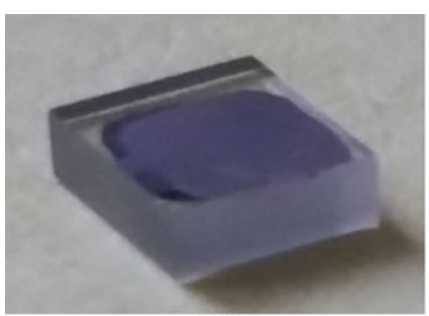

(a)

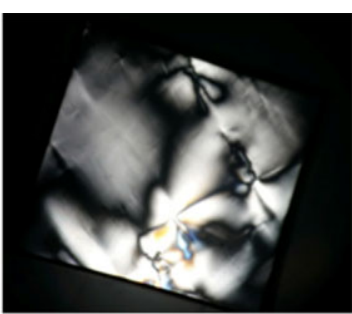

(b)
Figure 1 (a) Aluminium metalized IIa-Tech scCVD sample before mounting into the TCT setup. (b) Crossed-polarizers birefringence image of the $3 \times 3 \mathrm{~mm}^{2}$ sample.

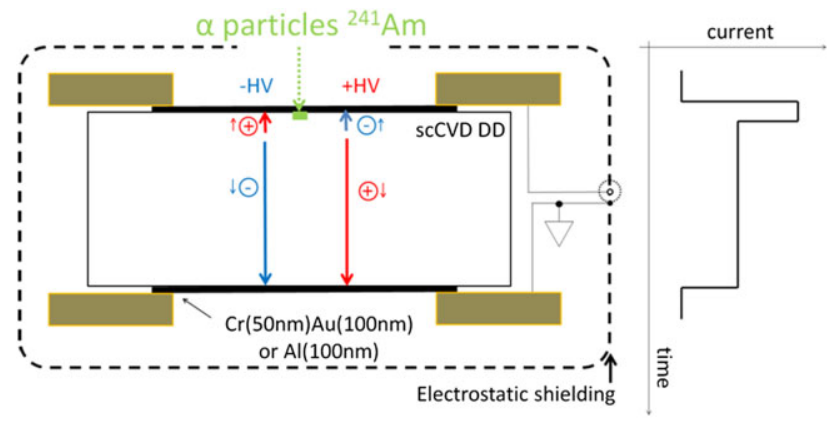

Figure 2 Principle of transient current technique (TCT) measurement using short-range $\alpha$-particles as excess charge carrier's generator. Right: schematic representation of the current induced, resulting from the carriers displacement in the sample, and providing insights of the space charge present in the bulk of the material. By varying the bias direction, the approach enables to evaluate whether defects are limiting hole or electron transports.

boards (pcbs). Pcbs, at the same time, provide the electrical contact to the read-out electronics and a collimation for $\alpha$-particles, to avoid single event upsets located at the vicinity the sample's electrodes (Fig. 2). A broad-band CIVIDEC C2 [9] amplifier was used for signal amplification $(40 \mathrm{~dB})$. Transient current waveforms were measured, stored and analyzed on a $2 \mathrm{GHz}$ bandwidth $(20 \mathrm{GS} / \mathrm{s})$ LeCroy oscilloscope. The DC bias was applied to the sample through an ORTEC 660 power supply. High voltage bias is supplied through a bias-T. More detailed description of the TCT set-up can be found in Ref. [7].

2.4 X-ray-induced photocurrents The sample has been characterized under continuous X-ray exposure from a mammography low energy tube. The X-ray tube is operated at $50 \mathrm{keV}$ with $0.5 \mathrm{~mA}$ current and the X-ray flux can be instantly turned off thanks to a manual lead shutter. The photocurrent induced in the sample is measured through an electrometer Keithley 6517A and recorded on a computer.

\section{Results and discussion}

3.1 Cathodoluminescence The CL spectrum of the sample is given in Fig. 3. It is dominated by the band-edge emissions in the UV region. The free exciton (FE) recombination assisted with TO, TA, or LO phonons was the only radiation observed in the bandgap energy region. The sample reveals a quite narrow $\mathrm{FE}_{\mathrm{TO}}$ peak, with a full width at half maximum (FWHM) of $3.3 \mathrm{meV}$, to be compared with the $2 \mathrm{meV}$ lowest reported value [10]. Up to $3 \mathrm{O}^{\mathrm{I}}$ phonon replica of the $\mathrm{FE}_{\mathrm{TO}}$ were observed as shown in the inset of Fig. 3. Such a luminescence spectrum is typical of high purity, high structural quality intrinsic diamond [11]. More specifically, the absence of boron-bound exciton recombinations [12] demonstrates that the boron acceptor concentration is below the CL detection limit, which is $1 \times 10^{13} \mathrm{~cm}^{-3}$ at liquid helium temperature.

In addition, we observe the luminescence from deep defects in the visible. The band having a zero phonon line 


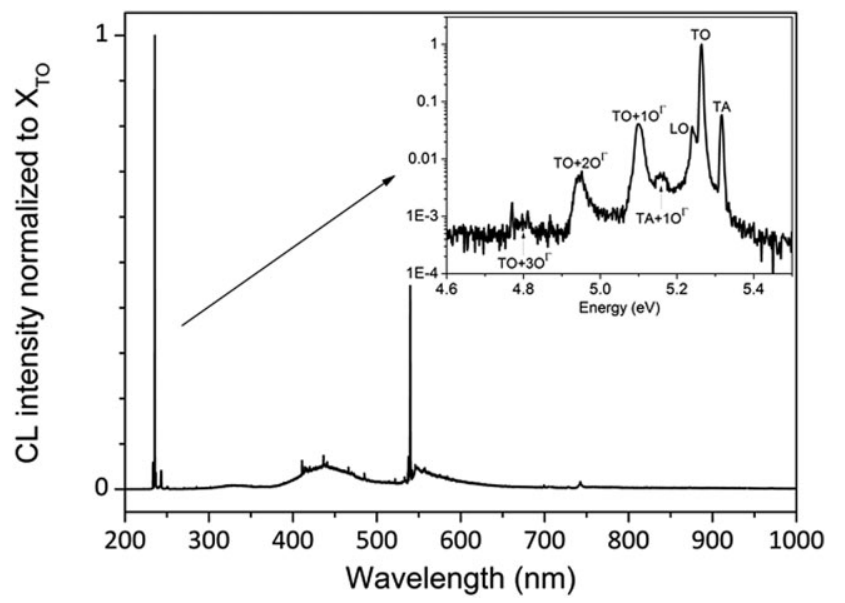

Figure 3 Cathodoluminescence spectrum of the IIa scCVD diamond sample at $8 \mathrm{~K}$. For clarity, the excitonic signals detected at the second, third, and fourth order of the diffraction grating were removed. In the inset, the intrinsic diamond emission shows the free exciton (FE) recombination assisted by TO, TA, and LO phonons and followed by several $\mathrm{O}^{\Gamma}$ phonon replica.

at $540 \mathrm{~nm}$ is the most important visible contribution. Its attribution is not straightforward according to the diamond deep defect database [13] and would require further investigations. A weak luminescence from the dislocation related A-band $(435 \mathrm{~nm})$ and the GR 1 center $(741 \mathrm{~nm})$ could also be detected. However, it is noticeable that no emission from the silicone-related center at $738 \mathrm{~nm}$ could be detected neither from the nitrogen-related center at $575 \mathrm{~nm}$. The CL analysis, thus, confirms the extremely low level of contamination by boron, nitrogen, and silicon residual impurities in the IIa scCVD diamond sample.

3.2 Transient current technique (TCT) A short range $(\sim 14 \mu \mathrm{m}) \alpha$-particle impinges into the tested sample through the entrance electrode. Due to the ionizing energy loss, cascades of excess e- $h$ pairs are created within the diamond bulk. After the thermalization process (a much shorter period than temporal resolution of the measurement set-up), meaning that electrons are located in the conduction band minima, and holes in the valence band maxima, e- $\mathrm{h}$ pairs are separated by the applied external electric field and start to drift and diffuse to the opposite electrodes, thus, inducing current on the metal electrodes. Depending on the direction of the applied electric field and taking into account short range $(14 \mu \mathrm{m})$ of $\alpha$-particles compared to the sample thickness $d(890 \mu \mathrm{m})$, we can assume that the major contribution to the induced current has only one type of carriers at once, thus, the charge transport parameters like drift velocity, mobility, lifetime, and others can be extracted for both charge carriers separately. Complete mathematical description of the formation of the transient currents can be found in diverse publications [14-18]. Here, since we want only to assess a high quality of the diamond material, complete mathematical description is dispensable.
In the ideal case of high purity material of parallel plate geometry, where the lifetime of the charge carriers, significantly exceeds the transient time $t_{\mathrm{r}}$ (defined as a time needed for the charge cloud to cross from the point of creation towards the opposite electrode, in our case $\sim 890 \mu \mathrm{m}$ ) and there is no internal space charge (e.g., due to the ionized impurities, or trapped charge), a constant current is observed in a short period of time $t_{\mathrm{r}}$ (Fig. 4) for both electron and hole majority drift. The shape of the TCT confirms constant currents thus constant velocity, thus, no observable space charge either in the bulk nor close to the contacts. This confirms the absence of space charge under the contacts, thus, their ohmic nature. Since both carriers contribute to the drift, a spike of minority carrier drift should be observed at the very beginning of the TC signal (Fig. 4). In practice this time $(\sim 100 \mathrm{ps})$ is shorter or comparable to the bandwidth of the measurements system, thus, this part of the TC signal is strongly suppressed. The integrals of current signals over the $t_{\mathrm{r}}$ gives the collected charge. If there is negligible trapping (no charge losses), the collected charge is equal to the generated charge and saturates quickly to a constant value, which is proportional to the $E_{\alpha} / \varepsilon_{\text {avg }}$, where $E_{\alpha}$ is $\alpha$-particle energy in $\mathrm{eV}$ and $\varepsilon_{\text {avg }}$ average energy needed to create e-h pair in diamond, values from $11.4 \mathrm{eV} / \mathrm{e}-\mathrm{h}$ to $16 \mathrm{eV} / \mathrm{e}-\mathrm{h}$, are frequently reported [19]. Such integrals are function of the detector bias voltage and are displayed in Fig. 5. Both electron and hole integrals saturate to the same value of $\sim 4.25 \times 10^{-10} \mathrm{Vs}$ already at weak electric field of $E \geq 0.2 \mathrm{~V} / \mu \mathrm{m}$, a strong indication of complete charge collection efficiency (CCE) even for sample thickness approaching $1 \mathrm{~mm}$.

In parallel plate geometry, with negligible trapping and space charge, a good approximation of the transient time, $t_{\mathrm{tr}}$ (the time needed for e or $\mathrm{h}$ cloud to cross the sample), is the FWHM of the TC signals. Thus, from a simple relation $d / t_{\text {tr }}$

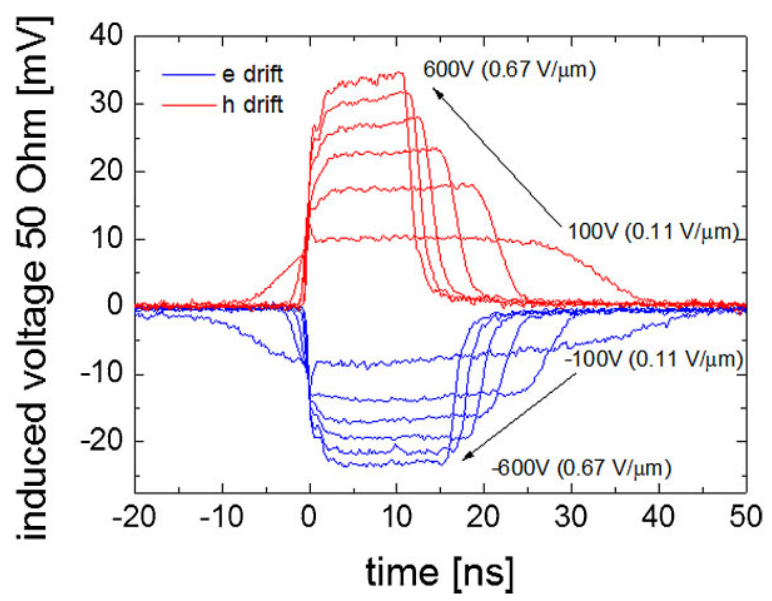

Figure 4 Transient current signals as a function of applied bias, (in red, majority of hole drift, in blue, majority of electron drift) induced by $5.486 \mathrm{MeV} \alpha$-particles within the IIa-Tech scCVD diamond sample metallized with parallel plate geometry $\mathrm{Al}$ contacts. 


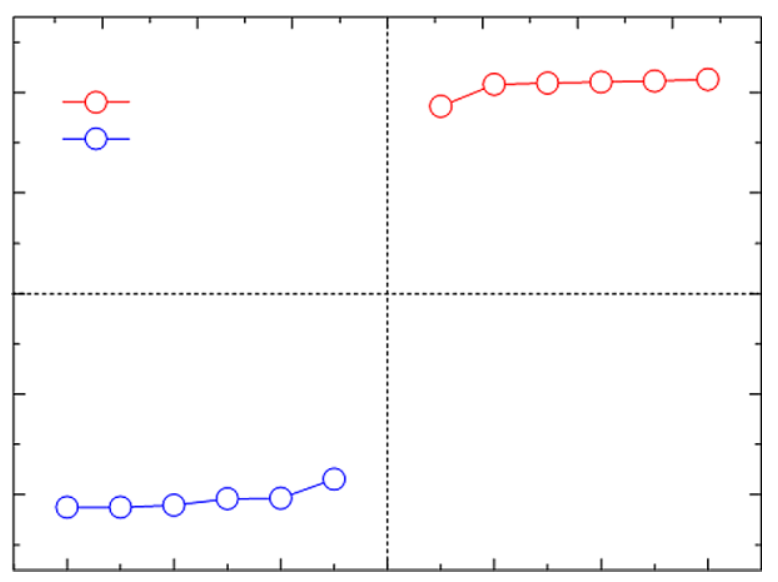

Figure 5 Integrals of TC signals from Fig. 4, values are directly proportional to the collected charge, quick saturation to the same value of $4.25 \times 10^{-10}$ Vs for both of e-h drift, at around $200 \mathrm{~V}$ $(0.22 \mathrm{~V} / \mu \mathrm{m})$, indicates full CCE.

( $d$ being the sample thickness), we can obtain the drift velocity of charge carriers. Drift velocity in $\langle 100\rangle$ direction with increasing detector bias (electric field) is displayed in Fig. 6 for electrons in blue, and holes in red. Additionally, two continuous lines are put on the graph: these are empirical fits to the experimental data of the drift velocity of scCVD diamonds from e6 supplier (about 20 samples measured) from [20]. The empirical formula used for the fit was first proposed by Coughey and Thomas in [21]

$$
\left.V=V_{\text {sat }}\left[\left(E / E_{\mathrm{c}}\right) /\left[1+\left(E / E_{\mathrm{c}}\right)^{\beta}\right)\right]^{1 / \beta}\right],
$$

where $V$ is the measured drift velocity, $E_{\mathrm{c}}$ and $\beta$ are the fitting parameters, $V_{\text {sat }}$ is the saturation drift velocity. The $E_{\mathrm{c}}$

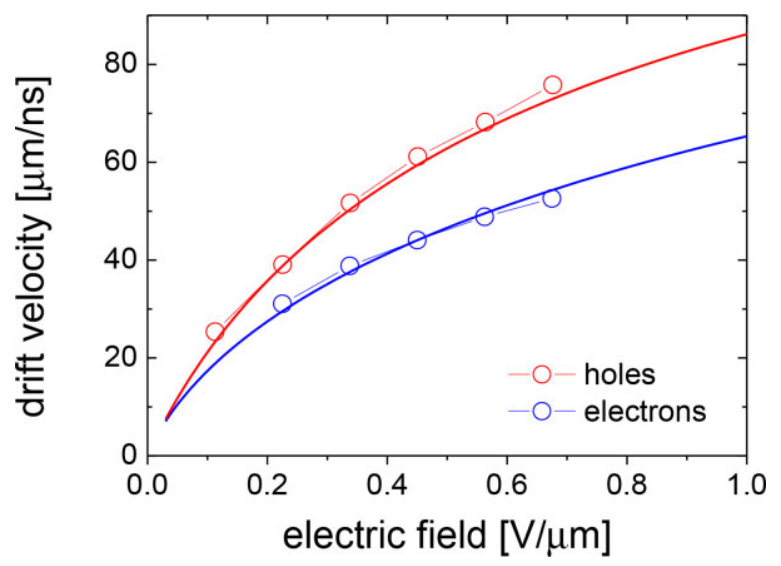

Figure 6 Measured drift velocity of electrons (in blue) and holes (in red) of IIa-Tech scCVD with increasing electric field. Open dots are for the experimental data of the IIa-Tech scCVD sample and lines are for empiritical fits from previous experimental data obtained on $\sim 20$ samples from ElementSix Ltd. [20].
Table 1 Fitting parameters for drift velocity of the scCVD diamonds from IIa-Tech and e6 suppliers.

\begin{tabular}{lcccr}
\hline & $E_{\mathrm{c}}(\mathrm{kV} / \mathrm{cm})$ & $\mu_{0}\left(\mathrm{~cm}^{2} / \mathrm{Vs}\right)$ & $V_{\text {sat }}(\mathrm{cm} / \mathrm{s})$ & \multicolumn{1}{r}{$\beta$} \\
\hline electrons & 5.779 & 4551 & $2.63 \times 10^{7}$ & 0.42 \\
& \pm 0.772 & \pm 500 & $\pm 0.2 \times 10^{7}$ & \pm 0.01 \\
holes & 5.697 & 2750 & $1.57 \times 10^{7}$ & 0.81 \\
& \pm 0.529 & \pm 70 & $\pm 0.14 \times 10^{7}$ & \pm 0.01 \\
\hline
\end{tabular}

parameter is related to the low field mobility, $\mu_{0}$ given by $\mu_{0}=V_{\text {sat }} / E_{\mathrm{c}}$.

As evidenced by Fig. 6, drift velocity data points of a IIa-Tech sample fit perfectly to the previously measured drift velocity characteristics of scCVD diamond. The fitting parameters of Eq. (1), obtained for e6 scCVD diamonds, are also valid for the IIa-Tech scCVD sample. They are given in Table 1.

The physical fit parameters of mobilities are in very good agreement with previously published values $[5,6]$. The saturation velocity for both, e and h, is higher than the reported values, but in good agreement with theoretical calculations [22].

A pulse height spectrum measured on the IIa-Tech diamond under 5.486 MeV $\alpha$-particles is presented in Fig. 7. A conventional nuclear spectroscopy chain was used for the measurement, whereas the broad-band amplifier is replaced by a charge sensitive preamplifier exhibiting a much better $\mathrm{S} / \mathrm{N}$ ratio with respect to broad-band. Although the time information is lost, due to the integration of the signal, the measurement of the collected charges (here, signal amplitude) is much more precise in this case. A single peak, corresponding to $\alpha$ energy of $5.486 \mathrm{MeV}$ is visible in the spectrum, with relative resolution, $\Delta E=\mathrm{FWHM} / x_{\mathrm{c}}$ of only $0.8 \%$. Such a low value of $\Delta E$ approaches that of the best quality e6 scCVDs commonly obtained on much thinner samples $(d<100 \mu \mathrm{m})$ [7]. During several hours of continuous measurement, no peak shift was observed. Both

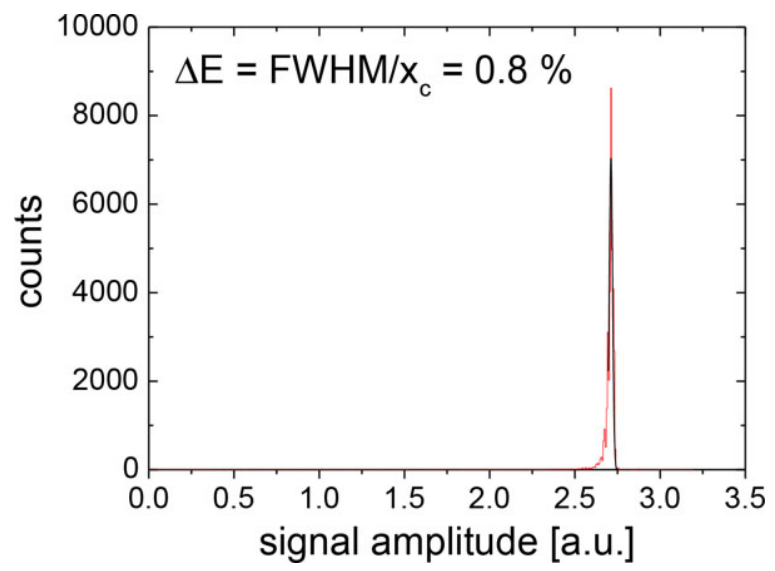

Figure 7 A pulse height spectrum of $5.486 \mathrm{MeV} \alpha$-particles (Am241 source) measured in vacuum with IIa-Tech scCVD diamond and charge sensitive electronics, here majority of holes drift. 


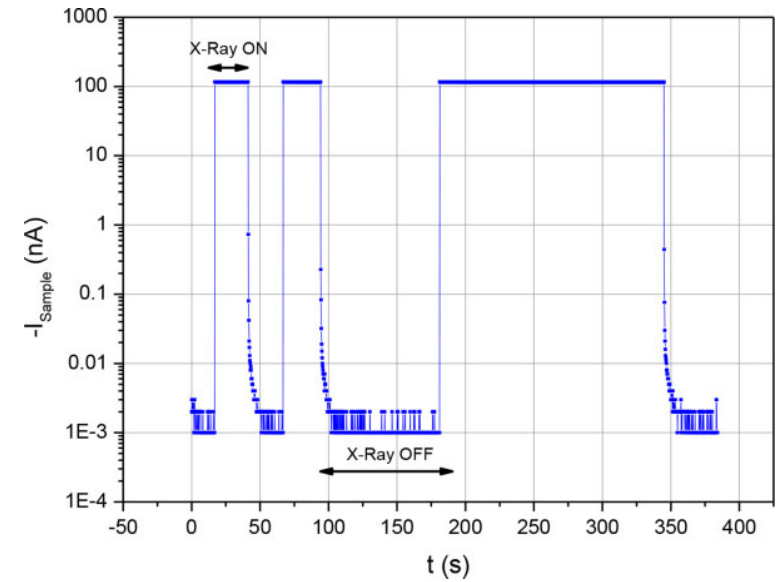

Figure 8 Sample electrical response under $50 \mathrm{keV}$ X-ray exposure.

observations indicate the high homogeneity of the charge collection efficiency for this IIa-Tech diamond sample, not only for time (negligible trapping, polarization) but also for the sample volume (absence of defects affecting the charge transport).

3.3 X-ray-induced current The sample is exposed to the X-ray flux at approximatively $1 \mathrm{~cm}$ of the external window. Part of the incoming energy is absorbed in the sample and $\mathrm{e}-\mathrm{h}$ pairs are created in the diamond. Here, the steady-state induced current is measured under a constant bias applied between the opposite faces of the sample and collecting the created carriers (Fig. 8).

Although conventional diamonds may exhibit an unstable response at the initial exposure due to deep or shallow level carrier progressive trapping [23] ("priming" or "overshoot," respectively), almost no effect is observed here. As the X-ray flux is repeatedly turned on and off to qualitatively assess the time response of the detector and its stability, there is no delay neither at the photocurrent to find a steady equilibrium or to reach background noise. Also, a very high signal to noise is observed with over 5 orders a magnitude between on and off photocurrents. Such very good rising and falling times of the signal depict the high quality of the IIa-Tech sample with remarkable stability under steady state $\mathrm{X}$-rays and high signal to noise.

4 Conclusions The stability of the pulse height spectra with respect to time is a good indication of the absence of space charge limited effects as observed in poor quality SC diamonds. Here, acquisition during several tens of hours demonstrated the very weak effects of carrier trapping and accumulation, further to be confirmed under mammography X-ray beam irradiation.

From our experiences, the quality is at least equal to the best quality scCVD commercially available. Ideally, more samples should be assessed shortly to probe the reproducibility of those performances from part-to-part. Since the test is not destructive, the approach can be tested on a regular basis for material inspection.

\section{References}

[1] J. F. Hochedez, J. Alvarez, F. D. Auret, P. Bergonzo, M. C. Castex, A. Deneuville, J. M. Defise, B. Fleck, P. Gibart, S. A. Goodman, O. Hainaut, J. P. Kleider, P. Lemaire, J. Manca, E. Monroy, E. Munoz, P. Muret, M. Nesladek, F. Omnes, E. Pace, J. L. Pau, V. Ralchenko, J. Roggen, U. Schühle, and C. Van Hoof, Diam. Relat. Mater. 10, 673-680 (2001).

[2] W. Adam, B. Bellini, E. Berdermann, P. Bergonzo, W. De Boer, F. Bogani, E. Borchi, A. Brambilla, M. Bruzzi, C. Colledani, J. Conway, P. d'Angelo, W. Dabrowski, P. Delpierre, J. Doroshenko, W. Dulinski, B. Van Eijk, A. Fallou, P. Fischer, F. Fizzotti, C. Furetta, K. K. Gan, N. Ghodbane, E. Grigoriev, G. Hallewell, S. Han, F. Hartjes, J. Hrubec, D. Husson, H. Kagan, J. Kaplon, C. Karl, R. Kass, M. Keil, K. T. Knöpfle, T. Koeth, M. Krammer, A. Logiudice, R. Lu, L. Mac Lynne, C. Manfredotti, R. D. Marshall, D. Meier, D. Menichelli, S. Meuser, M. Mishina, L. Moroni, J. Noomen, A. Oh, L. Perera, M. Pernicka, P. Polesello, R. Potenza, J. L. Riester, S. Roe, A. Rudge, S. Sala, M. Sampietro, S. Schnetzer, S. Sciortino, H. Stelzer, R. Stone, C. Sutera, W. Trischuk, D. Tromson, C. Tuve, P. Weilhammer, N. Wermes, M. Wetstein, W. Zeuner, and M. Zoeller, R.D.42 Collaboration, Nucl. Instrum. Methods A 511, 124-131 (2003).

[3] M. J. Guerrero, D. Tromson, M. Rebisz, C. Mer, B. Bazin, and P. Bergonzo, Diam. Relat. Mater. 13, 2046-2051 (2004).

[4] P. Bergonzo, D. Tromson, and C. Mer, Semicond. Sci. Technol. 18, S105-S112 (2003).

[5] J. Isberg, J. Hammersberg, E. Johansson, T. Wikströ m, D. J. Twitchen, A. J. Whitehead, S. E. Coe, and G. A. Scarsbrook, Science 297, 1670-1672 (2002).

[6] H. Pernegger, S. Roe, P. Weilhammer, V. Eremin, H. FraisKölbl, E. Griesmayer, H. Kagan, S. Schnetzer, R. Stone, W. Trischuk, D. Twitchen, and A. Whitehead, J. Appl. Phys. 97, 073704 (2005).

[7] M.Pomorski,E. Berdermann, A. Caragheorgheopol,M.Ciobanu, M. Kiš, A. Martemiyanov, C. Nebel, and P. Moritz, Phys. Status Solidi A 203 (12), 3152 (2006).

[8] IIa Technologies Pte. Ltd., www.2atechnologies.com

[9] Cividec Instrumentation, www.cividec.at/index.php?module= public.product_show\&id $=15 \&$ cat $=0$

[10] R. Sauer, Luminescence from Optical Defects and Impurities in CVD Diamond, Thin-Film Diamond I, Semiconductors and Semimetals Series, Vol. 76 (Elsevier, Academic Press, London, 2003).

[11] A. Tallaire, J. Achard, A. Secroun, O. De Gryse, F. De Weerdt, J. Barjon, F. Silva, and A. Gicquel, J. Cryst. Growth 291, 533 (2006).

[12] J. Barjon, T. Tillocher, N. Habka, O. Brinza, J. Achard, R. Issaoui, F. Silva, C. Mer, and P. Bergonzo, Phys. Rev. B 83, 073201 (2011).

[13] A. M. Zaitsev, Optical Properties of Diamond (Springer, Berlin, Heidelberg, New York, 2010).

[14] W. Shockley, J. Appl. Phys. 9, 635 (1938).

[15] S. Ramo, Proc. IRE 27 (9), 0096-8390 (1939).

[16] V. Eremin, N. Strokan, E. Verbitskaya, and Z. Li, Nucl. Instrum. Methods A 372, 388-398 (1996).

[17] Z. He, Nucl. Instrum. Methods A 463 (1-2), 250-267 (2001).

[18] M. Dris and T. Alexopoulos, arXiv:1406.3217v2 (2014). 
[19] M. Gabrysch, E. Marklund, J. Hajdu, D. J. Twitchen, J. Rudati, A. M. Lindenberg, C. Caleman, R. W. Falcone, T. Tschentscher, K. Moffat, P. H. Bucksbaum, J. Als-Nielsen, A. J. Nelson, D. P. Siddons, P. J. Emma, P. Krejcik, H. Schlarb, J. Arthur, S. Brennan, J. Hastings, and J. Isberg, J. Appl. Phys. 103, 064909 (2008)

[20] M. Pomorski, Electronic Properties of Single Crystal CVD 48 Diamond and Its Suitability for Particle Detection in Hadron
49 Physics Experiments, Ph.D. thesis, Universität Frankfurt am Main (2008).

[21] D. M. Caughey and R. E. Thomas, Proc. IEEE 55, 2192 (1967).

[22] D. K. Ferry, Phys. Rev. B 12 (6), 2361 (1975).

[23] P. Bergonzo, D. Tromson, C. Descamps, H. Hannita, C. Mer, N. Tranchant, and M. Nesladek, Diam. Relat. Mater. 16, 1038-1043 (2007). 